\title{
Rationale of Miyamoto's technique for reconstruction of tracheal carina
}

\author{
Kimihiro Shimizu, MD, PhD
}

Received: 26 April 2011

(C) The Japanese Association for Thoracic Surgery 2012

The article "Carinal resection and reconstruction for respiratory tumors using Miyamoto's technique" is an important contribution to the evaluation of Miyamoto's method outside Japan. Sayar and colleagues present interesting and exceptional results regarding the surgical resection of tracheobronchial tumors involving the tracheal carina. ${ }^{1}$

Carinal reconstruction to create a "neocarina" was first reported by Barclay and associates ${ }^{2}$ in 1957. At present, Barclay's method and the double-barrel method ${ }^{3}$ are the principal techniques used for carinal reconstruction. With the double-barrel method, a side-to-side anastomosis of the right and left bronchi is anastomosed end-to-end with the trachea. This approach results in high tension, however, especially at the side-to-side anastomosis. Additionally, adjusting the difference in the caliber of the trachea and the anastomosed bronchi is technically difficult. Consequently, this method is used mainly for resecting small segments of the airway and especially for simple resection of the carina without lung resection, as neither is vulnerable to high anastomotic tension.

With Barclay's method, the bronchus is anastomosed end-to-side with the lateral wall of the trachea or the bronchus after end-to-end anastomosis between the trachea and the contralateral bronchus. With this method, tension at the anastomosis is reduced such that longer segments of the airway can be resected. However,

This editorial refers to the article by Sayar et al. on pp. 90-96 of this issue of General Thoracic and Cardiovascular Surgery.

Department of Thoracic and Visceral Organ Surgery, Gunma University Graduate School of Medicine, 3-39-15 Showa-machi, Maebashi, Gunma 371-8511, Japan

Tel. +81-27-220-8245; Fax +81-27-220-8255

e-mail: kmshimizu@gmail.com blood flow at the lateral wall of the trachea or the bronchus may be impaired, and end-to-side anastomosis is technically demanding. Also, anastomosis with the trachea causes high tension if concomitant lung resection is performed because it is necessary to raise the remaining bronchus to the level of the trachea. Tension can be avoided by anastomosing the remaining bronchus to the left main bronchus, as is done in some cases; but again, this approach is technically highly demanding and places the left lung at greater risk.

With Miyamoto's method, the right bronchus is anastomosed end-to-side at the trimmed hole made at the anastomosis between the trachea and the left main bronchus. In the case of right tracheal sleeve pneumonectomy, anastomotic tension and impaired blood flow are highest at the right lateral aspect of the anastomosis. With Miyamoto's method, this site not only needs to be anastomosed but must also be reinforced with a right bronchus patch. However, because the patch site has a slight angle, these steps are technically easier than simple end-to-side anastomosis, as in Barclay's method. Furthermore, because the remaining bronchus is anastomosed only at the anastomosis between the trachea and left main bronchus, there is less tension on the final anastomosis than in Barclay's method, and there is no damage to the left main bronchus or to the retrograde blood supply of the end-to-end anastomosis.

Although I believe that theoretically Miyamoto's technique is the most reasonable carinal reconstruction method and it is technically easier, several questions remain. Yamamoto and coworkers presented 14 patients who were operated on using Miyamoto's technique in 2007. ${ }^{4}$ They reported that major anastomotic complications occurred in four patients (dehiscence in one, stenosis in three) and that all three patients with 
anastomotic stenosis had received induction chemotherapy. Additionally, three of five patients who received induction chemotherapy experienced anastomotic stenosis, whereas this was not the case in any of the nine patients who did not have induction chemotherapy. Based on these results, the authors concluded that the current indication for carinal resection after induction chemotherapy is tracheal sleeve pneumonectomy, which is a simpler procedure. By contrast, in the Sayar et al. series, four of six patients operated on using Miyamoto's technique received induction chemotherapy or chemoradiotherapy, and the other two patients received induction radiotherapy. None of these patients experienced anastomotic complications after surgery. As seen in the two bronchoscopic views (Figs. 5, 9 of the article) during the early postoperative phase ( 2 weeks) and late phase ( 9 years), the shape of the reconstructed carina is extremely natural, and there is no indication of anastomotic stenosis. To date, there is no clear evidence that induction chemotherapy or radiotherapy affects the healing of bronchial anastomoses, and there is no apparent explanation for the discrepancy between the results of these two reports.
The value of Miyamoto's method for patients receiving induction chemotherapy requires further evaluation with respect to prognosis as well as to morbidity and mortality. However, the article by Sayar et al. supports further use of Miyamoto's method for carinal reconstruction.

\section{References}

1. Sayar A, Solak O, Metin M, Celikten A, Çıtak N, Çetinkaya $\mathrm{E}$, et al. Carinal resection and reconstruction for respiratory tumors using Miyamoto's technique. Gen Thorac Cardiovasc Surg 2011;90-6.

2. Barclay RS, McSwan N, Welsh TM. Tracheal reconstruction without the use of grafts. Thorax 1957;12:177-80.

3. Mathey J, Binet JP, Galey JJ, Evrard C, Lemoine G, Denis B. Tracheal and tracheobronchial resections: techniques and results in twenty cases. J Thorac Cardiovasc Surg 1966;51: $1-13$.

4. Yamamoto K, Miyamoto Y, Ohsumi A, Imanishi N, Kojima F. Results of surgical resection for tracheobronchial cancer involving the tracheal carina. Gen Thorac Cardiovasc Surg 2007;55:231-9. 